\title{
Síntese e caracterização de catalisadores tipo HY/MCM-48 visando aplicações em processos FCC
}

\author{
Lima, E. R. N. ${ }^{1 *}$; Pedrosa ${ }^{2}$, A. M. G.; Souza, M. J. B. ${ }^{1,3}$ \\ 1 Programa de Pós-Graduação em Engenharia Química, Universidade Federal de Sergipe, São Cristóvão, SE, Brasil. \\ 2 Departamento de Química, Universidade Federal de Sergipe, São Cristóvão, SE, Brasil. \\ 3 Departamento de Engenharia Química, Universidade Federal de Sergipe, São Cristóvão, SE, Brasil. \\ *e-mail: elys_rayssa@hotmail.com
}

\begin{abstract}
Resumo
O objetivo deste trabalho foi estudar a síntese e caracterização de catalisadores micro-mesoporosos visando sua aplicação em processos de craqueamento realizado em leito fluidizado (FCC - Fluid Catalytic Cracking). Catalisadores micro-mesoporosos do tipo HY/MCM-48 em três proporções foram sintetizados através do método de mecanossíntese. Os catalisadores foram caracterizados por difração de raios-X e adsorção de $\mathrm{N}_{2}$ a $77 \mathrm{~K}$. Os difratogramas de raios-X indicaram que os catalisadores sintetizados apresentam os picos característicos da estrutura microporosa (zeólita HY) e da estrutura mesoporosa (MCM-48), mantendo assim as estruturas micro e mesoporosas após os procedimentos de síntese. As análises por adsorção de $\mathrm{N}_{2}$ revelaram que os materiais apresentaram expressivos valores de áreas superficiais específicas na faixa de 1100 a $2000 \mathrm{~m}^{2} \mathrm{~g}^{-1}$ e volume de poros na faixa de $0,26 \mathrm{a} 0,78 \mathrm{~cm}^{3} \mathrm{~g}^{-1}$.
\end{abstract}

\begin{abstract}
The aim of this work was to study the synthesis and characterization of micro- mesoporous catalysts for potential application in fluidized bed catalytic cracking processes (FCC - Fluid Catalytic Cracking). Micro mesoporous catalysts of HY/MCM -48 type at three different ratios were synthesized via mechanical synthesis method. The catalysts were characterized by X-ray diffraction and $\mathrm{N}_{2}$ adsorption at $77 \mathrm{~K}$. The $\mathrm{X}$ ray diffractograms indicated that the synthesized catalysts show characteristic peaks of the microporous structure (HY zeolite) and the mesoporous structure (MCM-48), thus maintaining the micro and mesoporous structures after the procedures of synthesis. The analysis by $\mathrm{N}_{2}$ adsorption showed that the materials have expressive values of specific surface areas in the range 1100 to $2000 \mathrm{~m}^{2} \mathrm{~g}^{-1}$ and a pore volume in the range 0,26 to $0,78 \mathrm{~cm}^{3} \mathrm{~g}^{-1}$.
\end{abstract}

Keywords (Palavras chaves): HY, MCM-48, micro-mesoporous materials.

\section{Introdução}

No craqueamento catalítico, a quebra das frações pesadas de hidrocarbonetos constituintes do petróleo se dá tanto pelo efeito térmico, quanto pela presença de um catalisador. Durante o processo de craqueamento catalítico ocorre uma desativação rápida do catalisador, motivada pela deposição de coque, aumentando assim o custo do processo. Isso geralmente era observado quando a reação se processava em grandes leitos catalíticos fixos. Devido a isto estudos culminaram com o desenvolvimento do processo de craqueamento realizado em leito fluidizado (FCC - Fluid Catalytic Cracking) [1].

A peneira molecular microporosa zeolítica do tipo $Y$ (Figura 1) é um dos principais componentes do catalisador do craqueamento, sendo amplamente empregada em países, como o Brasil, que craqueiam petróleos pesados de cadeia longa. As zeólitas demonstraram serem apropriadas para a realização de transformação catalítica dos hidrocarbonetos de interesse comercial nos campos da produção química e da refinaria de óleo, petroquímica. Esse sucesso se dá devido a possibilidade de modificação da força ácida do catalisador onde a acidez pode ser adaptada para a necessidade de uma reação, aumentando assim a conversão para um determinado produto [3]. 


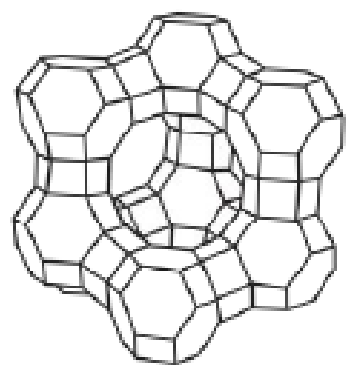

Figura 1: Estrutura da zeólita Y [2]

A peneira molecular MCM-48 é um material pertencente a família de materiais mesoporosos M41S, que apresenta uma rede de canais tridimensionais únicos que pode fornecer uma abertura altamente porosa com fácil e direto acesso para moléculas, assim facilitando inclusão ou difusão ao longo dos poros de canais sem o bloqueio destes [4]. A Figura 2 mostra um modelo para a estrutura mesoporosa cúbica do MCM$48[5]$.

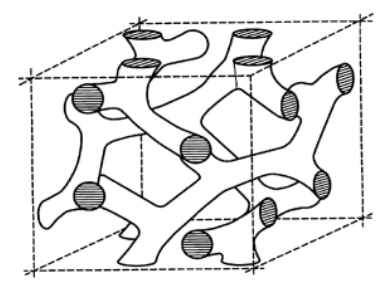

Figura 2: Modelo representativo para a estrutura cúbica do MCM-48 [6]

A combinação dos dois tipos de estrutura (microporosa zeolítica e mesoporosa do tipo MCM-48) pode gerar um catalisador que apresenta propriedades atrativas para usos em catálise, como é o caso do craqueamento catalítico em leitos fluidizados (FCC).

\section{Materiais e Métodos}

\subsection{Obtenção da zeólita HY}

A zeólita $H Y$ foi obtida através do processo de troca iônica com base no procedimento [7]. Para o procedimento foi utilizado 2,0 gramas de uma zéolita $\mathrm{NaY}$ comercial (CBV-100 da Zeolyst) e $250 \mathrm{~mL}$ de solução de cloreto de amônio a $1 \mathrm{~mol} \mathrm{~L}^{-1}$. O sistema de troca iônica foi mantido sob agitação constante a $80^{\circ} \mathrm{C}$ por 2 horas. A solução 1 resultante da troca iônica foi filtrada a vácuo e lavada com cerca de $1 \mathrm{~L}$ de água deionizada para remover os íons amônio $\left(\mathrm{NH}_{4}{ }^{+}\right)$não trocados e íons cloreto $\left(\mathrm{Cl}^{-}\right)$. Para a identificação dos íons cloreto no meio aquoso foram utilizadas algumas gotas de nitrato de prata $0,2 \mathrm{~mol} \mathrm{~L}^{-1}$.

A lavagem foi cessada quando não se observou mais a precipitação dos íons em meio aquoso. Depois das etapas descritas anteriormente, a amostra foi submetida a mais um processo de troca iônica. Ao final do processo, a amostra foi colocada em estufa a $100^{\circ} \mathrm{C}$ por 2 horas para secagem e depois submetida à calcinação em um forno mufla a $450^{\circ} \mathrm{C}$ com taxa de aquecimento de $10^{\circ} \mathrm{C} \mathrm{min}^{-1}$ por 2 horas, liberando assim a amônia e obtendo-se a forma HY. As etapas da síntese são mostradas na Figura 3.

\subsection{Síntese do MCM-48}

A síntese do MCM-48 foi feita pelo método hidrotérmico, empregando uma metodologia adaptada [8], [9] e [10]. Todas as etapas realizadas estão descritas na Figura 4. As razões molares dos precursores foram obtidas com base em cálculos estequiométricos a fim de se obter um gel de síntese com a composição molar de $0,55 \mathrm{CTMABr}: 0,25 \mathrm{Na}_{2} \mathrm{O}: 1,0 \mathrm{SiO}_{2}: 101,4 \mathrm{H}_{2} \mathrm{O}$. A primeira etapa consistiu na dissolução do hidróxido de sódio em água deionizada (solução 2). Em seguida adicionou-se o brometo de cetiltrimetilamônio (CTMABr) como direcionador de estrutura e manteve-se o sistema sob agitação por 30 minutos e aquecimento a $40^{\circ} \mathrm{C}$ formando uma solução 3. Logo após adicionou-se vagarosamente o tetraetilortosilicato (TEOS), como fonte de silício.

Para o gel de síntese obtido foi medido o $\mathrm{pH}$ e corrigido com gotas de uma solução de ácido acético $30 \%(\mathrm{v} / \mathrm{v})$, até $\mathrm{pH} 11$, logo após foi colocado em um vaso de teflon e autoclave de aço inox para tratamento hidrotérmico em estufa, a $120^{\circ} \mathrm{C}$, por um período de 24 horas. Posteriormente, a amostra resultante foi filtrada a vácuo e lavada com água deionizada e seca a $100^{\circ} \mathrm{C}$ por 2 horas em estufa, e calcinada em forno mufla a $450^{\circ} \mathrm{C}$ por 2 horas, com uma taxa de aquecimento de $10^{\circ} \mathrm{C} \min ^{-1}$.

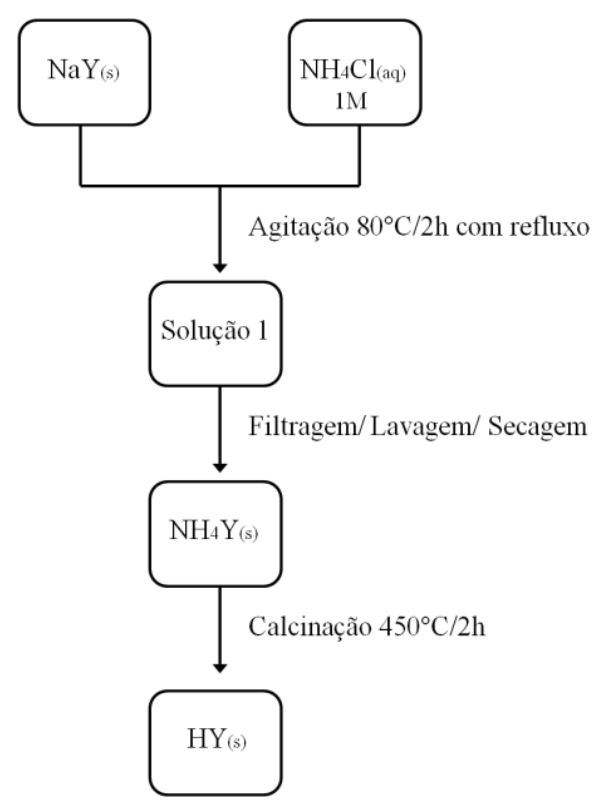

Figura 3: Etapas da síntese da zeólita HY 


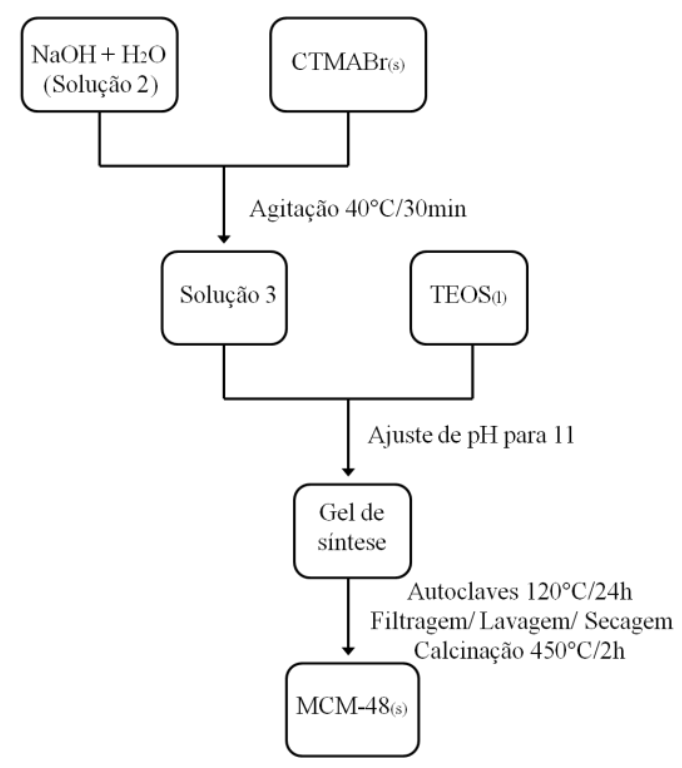

Figura 4: Etapas da síntese do material mesoporoso MCM-48

\subsection{Síntese do HY/MCM-48}

A síntese do catalisador compósito HY/MCM-48 foi realizada pelo método da mecanossíntese, empregando um procedimento adaptado [11], onde proporções com massas pré-estabelecidas, considerando o teor de umidade dos componentes microporoso $\mathrm{HY}$ e mesoporoso MCM-48, foram misturadas através da moagem em um almofariz com pistilo durante 20 minutos até homogeneização uniforme.

A mistura foi realizada em quatro etapas: uma quantidade equivalente a $1 / 4$ da massa do material microporoso e $1 / 4$ do material mesoporoso (previamente pesados na proporção estabelecida) foram colocados no almofariz e submetidos à mistura manual contínua durante 5 minutos. Este procedimento foi repetido por mais de três vezes, sendo que a cada etapa a massa dos materiais microporoso e mesoporoso era adicionada sobre o material já misturado contido no almofariz. As proporções mássicas utilizadas da zeólita HY e do material mesoporoso MCM-48 estão descritas na Tabela 1.

Tabela 1: Proporções da zeólita HY e do MCM-48 nos materiais micromesoporosos do tipo HY/MCM-48

\begin{tabular}{c|c|c|c}
\hline \multirow{2}{*}{ Proporção } & \multicolumn{2}{|c|}{ Percentual mássico } & \multirow{2}{*}{$\begin{array}{c}\text { Notação para } \\
\text { HY/MCM-48 }\end{array}$} \\
\cline { 2 - 3 } & HY & MCM-48 & 25HY/75MCM-48 \\
\hline $1: 3$ & $25 \%$ & $75 \%$ & $50 \mathrm{HY} / 50 \mathrm{MCM}-48$ \\
$3: 1$ & $50 \%$ & $50 \%$ & $75 \mathrm{HY} / 25 \mathrm{MCM}-48$ \\
\hline
\end{tabular}

2.4 Caracterização

\subsection{1 - Difratogramas de raios- $X$}

Através dessa técnica é possível obter informações sobre as fases presentes nos catalisadores. Para os catalisadores microporoso (HY) e mesoporoso (MCM48) foram investigadas as fases presentes e para o catalisador HY/MCM-48 foi investigada a manutenção das estruturas microporosas e mesoporosas após as etapas de síntese.

As análises de DRX foram realizadas através do método do pó em um equipamento Shimadzu XRD6000 com fonte de radiação $\mathrm{Cu}$, voltagem de 40 $\mathrm{kV}$, corrente de $30 \mathrm{~mA}$, velocidade de varredura $0,5^{\circ}$ $\mathrm{min}^{-1}$. Para o material $\mathrm{HY}$, foi utilizado um passo de $0,02^{\circ}$ graus e uma faixa angular $(2 \theta)$ de 5 a $55^{\circ}$, para a amostra de MCM-48 a faixa angular $(2 \theta)$ foi de 1 a $10^{\circ}$ e um passo de $0,01^{\circ}$. Os DRX dos materiais compósitos foram realizados em um equipamento Rigaku DMAX100 à $40 \mathrm{kV}$ e $40 \mathrm{~mA}$ em uma faixa de varredura angular de 1 a $60^{\circ}$ com passo de $0,02^{\circ}$.

\subsection{2 - Adsorção de $\mathrm{N}_{2}$ a $77 \mathrm{~K}$}

As análises de adsorção de $\mathrm{N}_{2}$ a $77 \mathrm{~K}$ foram realizadas em um equipamento da Quantachrome modelo NOVA 1200e. As áreas superficiais específicas foram obtidas pelo método BET (Brunauer-Emmett-Teller) na faixa de $P / P_{o}$ de 0,05 a 0,20 e os volumes de poros pelo método BJH (Barrett-Joyner-Halenda).

\section{Resultados e Discussão}

A Figura 5 mostra o difratograma de raios-X da zeólita HY. Os picos de difração observados podem ser atribuídos a estrutura do tipo FAU da zeólita $H Y$, caracterizada por reflexões intensas em $2 \theta$ igual a $6,4^{\circ}$, $23,76^{\circ}$ e $15,78^{\circ}$, conforme descritos na literatura [12] e [13]. Os demais picos presentes também são atribuídos a zeólita com estrutura do tipo FAU e com alto grau de pureza.

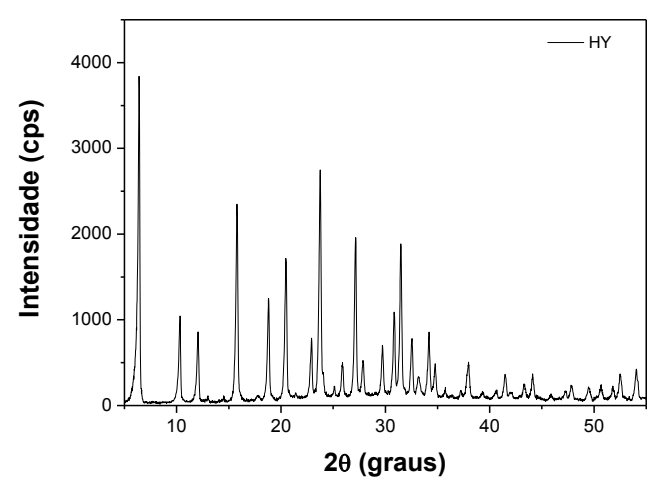

Figura 5: Difratograma de raios-X da zeólita HY

O difratograma de raios- $X$ do mesoporoso MCM-48 é mostrado na Figura 6. De acordo com a Figura 6 é possível observar os picos em região de baixo ângulo referente aos planos de reflexão (211) e (220), 
localizados em $2 \theta$ igual $2,44^{\circ}$ e $2,71^{\circ}$, e um conjunto de picos de baixa intensidade: (321), (400), (420), (332), (422) e (431), que são característicos de uma estrutura cúbica, comum a materiais MCM-48. Esses planos estão de acordo com vários difratogramas relatados na literatura para esse tipo de material [14], [15], [16] e [17].

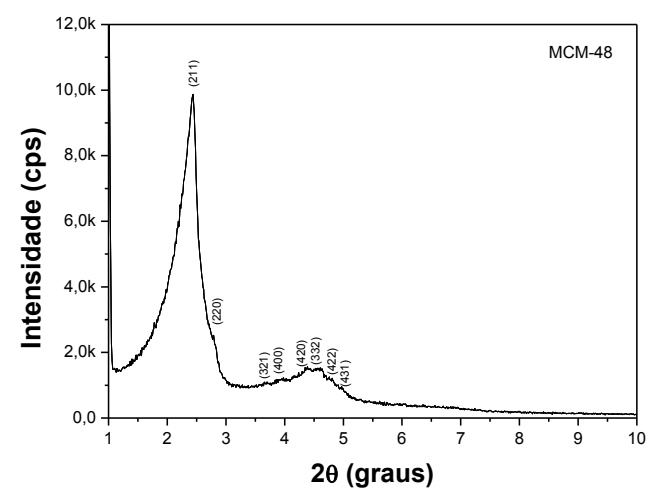

Figura 6: Difratograma de raios-X do mesoporoso MCM-48

Na Figura 7 têm-se os difratogramas de raios- $X$ dos catalisadores micro-mesoporosos do HY/MCM-48 em três proporções (25HY/75MCM-48; 50HY/50MCM-48; $75 \mathrm{HY} / 25 \mathrm{MCM}-48)$. Os difratogramas apresentaram picos de difração característico do $\mathrm{HY}$ para o catalisador $25 \mathrm{HY} / 75 \mathrm{MCM}-48$ em ângulo $2 \theta=6,2^{\circ}$; $23,64^{\circ}$ e $14,98^{\circ}$, para o catalisador $50 \mathrm{HY} / 50 \mathrm{MCM}-48$ em ângulo $2 \theta=6,46^{\circ} ; 23,86^{\circ}$ e $15,72^{\circ}$, e para o catalisador $75 \mathrm{HY} / 25 \mathrm{MCM}-48$ em ângulo $2 \theta=6,68^{\circ}$; $23,94^{\circ}$ e $14,12^{\circ}$. Todos os difratogramas dos catalisadores apresentaram picos de difração devido ao MCM-48 em baixos ângulos, confirmando assim que após 0 processo de mecanossíntese houve a manutenção da estrutura do MCM-48 no catalisador micro-mesoporoso.

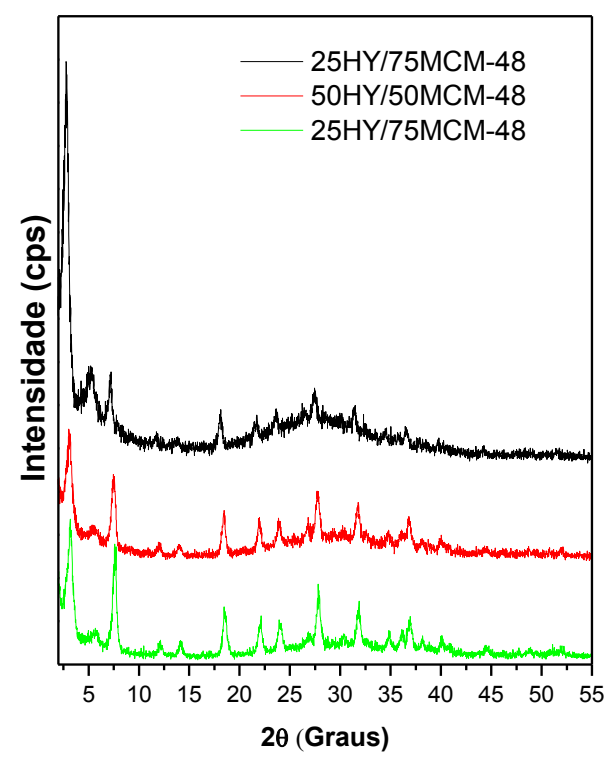

Figura 7: Difratograma de raios-X dos catalisadores micromesoporosos do tipo HY/MCM-48
De acordo com a Figura 7 é observado que a intensidade dos picos relativos ao $\mathrm{MCM}-48$ é levemente diminuída em relação ao MCM-48 puro (Figura 6), refletindo dessa forma na diminuição do grau de ordenamento da estrutura (Tabela 2).

É possível observar que nas amostras relativas ao HY/MCM-48 ocorreu deslocamento do pico para ângulos um pouco menores em $2 \theta$, observado através do ligeiro aumento nos valores do parâmetro mesoporoso nas amostras. No entanto, esse aumento no valor do parâmetro mesoporoso não é significativo, já que os picos característicos do MCM-48 estão presentes, mostrando dessa forma que os mesocanais são mantidos [18] e [19].

$\mathrm{Na}$ Tabela 2 é mostrado os valores do parâmetro mesoporoso $\left(\mathrm{a}_{0}\right)$ e o grau de ordenamento $(\mathrm{GO})$ para o MCM-48 nos catalisadores 25HY/75MCM-48, $50 \mathrm{HY} / 50 \mathrm{MCM}-48$ e $75 \mathrm{HY} / 25 \mathrm{MCM}-48$. O GO para o MCM-48 foi calculado com relação ao pico (211) do MCM-48 puro e comparado a intensidade do pico nas demais amostras.

Tabela 2: Principais parâmetros estruturais MCM-48 nos catalisadores HY/MCM-48

\begin{tabular}{|c|c|c|c|c|c|}
\hline Catalisador & $\begin{array}{c}\text { Índice } \\
\text { de } \\
\text { Miller } \\
\text { (hkl) }\end{array}$ & $\begin{array}{c}2 \theta \\
\text { (graus) }\end{array}$ & $\begin{array}{l}d_{211} \\
(n m)\end{array}$ & $a_{0}(n m)$ & $\begin{array}{l}\text { GO } \\
\text { (\%) }\end{array}$ \\
\hline MCM-48 & (211) & 2,44 & 3,62 & 8,87 & 100 \\
\hline $\begin{array}{c}25 \mathrm{HY} / 75 \mathrm{MCM}- \\
48\end{array}$ & $(211)$ & 2,42 & 3,65 & 8,94 & 97 \\
\hline $\begin{array}{c}50 \mathrm{HY} / 50 \mathrm{MCM}- \\
48\end{array}$ & (211) & 2,40 & 3,68 & 9,02 & 24 \\
\hline $\begin{array}{c}75 \mathrm{HY} / 25 \mathrm{MCM}- \\
48\end{array}$ & $(211)$ & 2,43 & 3,64 & 8,91 & 31 \\
\hline
\end{tabular}

Através das análises de adsorção de nitrogênio se observou que as amostras HY, MCM-48, 25HY/75MCM-48, 50HY/50MCM-48 e 75HY/25MCM48 apresentaram valores de áreas superficiais específicas BET de 587, 1241, 1124, 1996, $1098 \mathrm{~m}^{2} \mathrm{~g}^{-1}$ e volumes de poros de 0,$225 ; 0,693 ; 0,256 ; 0,780$ e 0,319 , respectivamente.

\section{Conclusões}

Os difratogramas de raios-X dos catalisadores 25HY/75MCM-48, 50HY/50MCM-48 e 75HY/25MCM48 mostraram a presença conjunta das fases microporosa (HY) e mesoporosa (MCM-48). Os catalisadores micro-mesoporosos apresentaram um pequeno aumento no parâmetro do mesoporo e uma significativa redução no grau de ordenamento hexagonal em relação ao MCM-48 puro. Os catalisadores apresentaram significativos valores de 
áreas superficiais específicas e volumes de poros na faixa de 1100 a $2000 \mathrm{~m}^{2} \mathrm{~g}^{-1}$ e 0,26 a $0,78 \mathrm{~cm}^{3} \mathrm{~g}^{-1}$. Tais características estruturais podem tornar esses catalisadores potencialmente atrativos para processos de conversão catalítica de hidrocarbonetos, como processos de FCC.

\section{Agradecimentos}

Os autores agradecem ao Programa de PósGraduação em Engenharia Química da UFS (PEQ/UFS) e ao Conselho Nacional de Desenvolvimento Científico e Tecnológico (CNPq) pela bolsa concedida.

\section{Referências}

[1] BIONDA, K. S. et al. Fuel Proc. Tech., v. 64, p. 107$115,2000$.

[2] BAERLOCHER, C.H., et al. Atlas of Zeolite Structure Types. 6 ed. Amsterdam: Elsevier, 2007.

[3] BORGES, L. D. Preparação, Caracterização e Atividade Catalítica de Zeólitas Y Desaluminizadas: Investigação das Interações Fluido-Sólido. $134 \mathrm{f}$. Dissertação (Mestrado em Química) - Instituto de Química, Universidade de Brasília, Distrito Federal, 2011.

[4] YATES, T. J. V., et al. Chem. Phy. Lett., v. 418, p. 540-543, 2006

[5] KIM, S., et al. J. Phy. Chem. B, v.109, p.628, 2008.

[6] ROTH, W. J.; SEWELL, N. J. U. S. Patent 6, 096, $288,2000$.

[7] MACHADO, S. W. M. Desenvolvimento de materiais híbridos micro-mesoporosos contendo terras raras para utilização no craqueamento de frações de petroléo.150 f. Dissertação (Mestrado em Química)Programa de Pós-Graduação em Química, Universidade Federal de Sergipe, Sergipe, 2015.
[8] JUN, S., et al. Microp. Mesop. Mat., v. 41, p. 199$127,2000$.

[9] DOYLE, A; HODNETT, B. K. Microp. Mesop. Mat., v. 63 , p. 53-57, 2003.[10] BOOTE, B., et al. Chem. Com., v. 43, p. 4543- 4545, 2007.

[10] BOOTE, B., et al. Chem. Comm., v. 43, p. 45434545, 2007

[11] SILVA, S. C. G. S. Síntese de materiais compósitos micromesoporosos visando a captura de $\mathrm{CO}_{2}$. 200f. Tese (Doutorado em Ciência e Engenharia de Materiais)- Programa de Pós-Graduação em Ciência e Engenharia de Materiais, Universidade Federal de Sergipe, Sergipe, 2015.

[12] PEdRosA, A. M. G., et al. Mat. Res. Bull., v. 41, p. 1105-1111, 2006.

[13] RAMLI, N. A. S.; AMIN, N. A. S. App. Cat. B: Env., v. 163, p. 487-498, 2015.

[14] SOUZA, M. J. B., et al. J. Ther. Anal. Cal., v. 79, p. 493-497, 2005.

[15] ZHAO, W., et al. Mat. Res. Bull., v. 40, p. 17751780, 2005.

[16] ZHAO, D.; WAN, Y. The synthesis of mesoporous molecular sieves. 3 ed., chap. 8, Amsterdam: Elsevier, 2007.

[17] HUANG, L., et al. Microp. Mesop. Mat., v. 111, p. 404-410, 2008.

[18] KRESGE, C. T.; ROTH, W. J. Chem. Soc. Rev., v. 42, p. 3663-3670, 2013.

[19] RÊGO, A. C. B., et al. Scien. Plen. v.12, n. 5, p. 1 10, 2016. 